\section{PM0-106 THE ROLE OF ENDOSCOPIC ULTRASOUND IN IDENTIFYING CHRONIC PANCREATITIS: A COMPARISON PATHOLOGICAL SPECIMENS}

doi:10.1136/gutjnl-2012-302514b.106

${ }^{1} \mathrm{~L} M$ Quinn, ${ }^{*}{ }^{2} \mathrm{M}$ Johnstone, ${ }^{1,2} \mathrm{R}$ Sutton, ${ }^{1} \mathrm{~S}$ Sarkar. ${ }^{1}$ Royal Liverpool University Hospital, Liverpool, UK; ${ }^{2}$ Liverpool NIHR Pancreas Biomedical Research Unit, University of Liverpool, Liverpool, UK

Introduction Imaging diagnostic criteria for chronic pancreatitis (CP) have long been debated. Patients with CP frequently undergo Endoscopic Ultrasound (EUS) as part of their diagnostic pathway, but it is unclear if should it be used as an adjunct for diagnosis or to simply exclude more sinister pathology.

Aim To assess the effectiveness of EUS in diagnosis in patients with CP when comparing resection specimens as the gold standard.

Methods All patients who had undergone EUS within 1 year prior to pancreatic resection between 2008 and 2011 at the Royal Liverpool University Hospital were identified from a prospectively maintained database. Resection specimens were compared to EUS and fine needle aspiration (FNA) findings. Agreement was assess using $\kappa$ statistic and groups were compared using $\chi^{2}$

Results In the cohort of 93 patients (42\% male; median [IOR] age 60 [51-72]) that underwent pancreatic resection, 60 (65\%) patients had a clinical suspicion of CP. In the resection specimens CP was determined in 47 (51\%) cases; 29 of which had CP alone and 18 with associated neoplasia. EUS correctly reported CP in $28(60 \%)$ patients; 15 alone \& 13 with a mass or cyst. EUS falsely identified three as $\mathrm{CP}$, and missed the diagnosis in 19 thus giving a sensitivity of $60 \%$ and specificity of $93 \%$ (positive predictive value $90 \%$; negative predictive value $70 \%$ ). Overall agreement EUS and resection histology was moderate $(\kappa 0.53)$. In the 29 resection specimens where CP was the sole histological diagnosis, there was good agreement with EUS which identified this as the sole pathology in $18(62 \%)$ cases ( $\kappa 0.62)$. However, in these cases, EUS reported CP in association with other pathology incorrectly in $11 / 29$ (38\%) of cases, querying neoplasia in $9 / 29(31 \%)$. The sensitivities of radial EUS (50) \& linear EUS with FNA (43) were similar (54\% \& $65 \%$; $\mathrm{p}=0.47$ ). FNA cytology only confirmed the diagnosis of CP in $2 / 11$ (15\%) were CP was the sole diagnosis, both of which were already been identified on sonography. The EUS detection of neoplasia in patients with CP was only $53 \%(9 / 17)$.

Conclusion Overall EUS only showed moderate agreement with histology in diagnosing $\mathrm{CP}$, which improved when CP was the sole diagnosis. There was no difference between linear and radial EUS, and FNA did not enhance the diagnosis of CP. This study shows that in context of CP, the EUS diagnosis of a concurrent neoplasia remains to prove difficult. Further detailed evaluation between clinical course, histological and EUS findings may be helpful.

Competing interests None declared.

\section{PM0-107 ROLE OF ERCP IN THE MANAGEMENT OF ACUTE GALLSTONE PANCREATITIS-A REVIEW OF CURRENT PRACTICE}

doi:10.1136/gutjnl-2012-302514b.107

M Johnstone, P Marriott, ${ }^{*}$ J Royle, E Hepburn, A Torrance, C Richardson, A Patel, D Bartlett, T Pinkney; On behalf of the West Midlands Research Collaborative, Birmingham, UK

Introduction Cholecystectomy is the preferred definitive treatment to prevent further episodes of gallstone pancreatitis, if patients have clear bile ducts and are suitable for surgery. Endoscopic retrograde cholangio-pancreatography (ERCP) is utilised to either clear the biliary tree of gallstones prior to operation or as definitive treatment in those deemed unsuitable for surgery. We aimed to determine how ERCP is being utilised, its effect on recurrent pancreatitis and readmission rates.

Methods A multi-centre retrospective review was performed of patients presenting with their first episode of gallstone pancreatitis between 2006 and 2008. All patients with confirmed biochemical diagnosis of pancreatitis plus a radiological diagnosis of gallstones were included. Data were collected on demographics, ERCP, operative management and readmissions. Groups were compared using $\chi^{2}$ and medians using Mann-Whitney $U$.

Results 523 patients were identified (36\% male, median age 63) in seven acute hospital trusts. 166 (32\%) underwent ERCP with 20 (13\%) having a failed procedure. 127 (77\%) underwent sphincterotomy with the common bile duct being clear in $80 \%$ (133). 28 $(17 \%)$ were performed within $72 \mathrm{~h}$ of admission and 105 (63\%) were performed during the index admission. There was no effect on the timing of ERCP in the likelihood of the procedure failing to be completed (median time 10 vs 10.5 days). 364 patients underwent cholecystectomy with $29 \%$ undergoing ERCP prior to surgery. ERCP was performed as the definitive procedure in 58 patients; median age 80 years compared to 58 years in those undergoing cholecystectomy ( $p$ two patients died following successful ERCPs; one 87 year old of Clostridium difficile colitis and one 81 year old of pancreatitis. There was one episode of post ERCP pancreatitis, and one bleed that resolved spontaneously. An additional 5 patients developed a second episode of pancreatitis subsequent to their ERCP. Sphincterotomy had not been performed in three of these cases $(p=0.08)$. ERCP with the index admission was the only significant factor to reduce recurrent pancreatitis rates (OR $0.092 \quad(0.013-0.673) ; \mathrm{p}<0.01$ ) however it failed to reduce the risk of readmission (OR 0.704 (0.39-1.26); $\mathrm{P}=0.24)$, which was only achieved by cholecystectomy within the index admission (OR 0.06 (0.01-0.4700); $p<0.01$ ).

Conclusion ERCP is currently being used as definitive treatment for gallstone pancreatitis in selected elderly patients. In our series ERCP had an acceptable rate of morbidity associated with the procedure, and was shown to significantly reduce the rate of recurrent pancreatitis. However, overall readmission rates were not improved by ERCP, with this only being achieved by cholecystectomy during the index admission.

Competing interests None declared.

\section{PMO-108 DIAGNOSIS OF PANCREATIC SOLID LESIONS WITH EUS AND FNA: A REPORT OF ACCURACY}

doi:10.1136/gutjnl-2012-302514b.108

S R Kadri, ${ }^{*}$ K Law, M James, K Ragunath, G Aithal. Department of Gastroenterology, Nottingham University Hospital, Nottingham, UK

Introduction Endoscopic ultrasound-guided fine needle aspiration (EUS-FNA) is an accurate method for cytological confirmation of pancreatic lesions, but it is unclear whether its diagnostic accuracy is affected by it's size, location, or size of needles used for biopsy. Our aim was to investigate the accuracy of EUS-FNA for suspected pancreatic lesions in relation to these factors, especially to the size of lesions, size of the needle used for biopsy and number of passes.

Methods In this tertiary referral centre, EUS-FNAs for 186 solid pancreatic lesions from 1 April 2010 to 31 March 2011 were evaluated retrospectively. Size of the lesion, site of the lesions were obtained as well as the information on size of the needle used to take biopsy and number of passes made and adequacy of the sample obtained.

Results 87 males and 85 females had 186 EUS FNA for solid pancreatic lesions.71\% (123/172) of then were aged between 51 and 80 years. 79 lesions were in the head of the pancreas alone. Information on adequacies for sampling was available for 149 procedures. Overall adequacy of EUS-FNA sampling was 76\% (114/149). 
Adequacy for the sample for lesions $<10 \mathrm{~mm}$ was $77 \%(10 / 13)$ and for more than $10 \mathrm{~mm}$ was $68 \%(104 / 153)$; those for lesions in the head was $70 \%(55 / 79)$ and for rest of the pancreas was $75 \%(59 / 79)$. Success rate for single, two, three and four passes made to obtain sample were $58 \%, 75 \%, 70 \%$ and $80 \%$ respectively. Accuracies for 19, 22 and 25-gauge needle sampling were $67 \%, 76 \%$ and $85 \%$ respectively.

Conclusion EUS-FNA has high accuracy in the evaluation of suspected pancreatic lesions regardless of its size, location of the lesion. It was useful also in confirming small pancreatic lesions that were $<10 \mathrm{~mm}$. 25-gauge needle produced best tissue yield out of all the types of the needles used for sampling.

Competing interests None declared.

\section{PM0-109 IGG4 RELATED AUTOIMMUNE DISEASE-EXPERIENCE FROM NORTH EAST OF ENGLAND}

doi:10.1136/gutjnl-2012-302514b.109

S Chatterjee, ${ }^{*} \mathrm{~K}$ Oppong, M Nayar. HPB Unit, Freeman Hospital, Newcastle upon Tyne, UK

Introduction IgG4 related disease is now well recognised as a multisystem disease. This condition, originally discovered in Japan in 1995 is now being increasingly recognised in the Western World. Apart from diagnosis, treatment can also present as a challenge in a small group of patients. We report our experience from a tertiary referral hospital in North-East England.

Methods Data were obtained from retrospective case note review from 2005 to 2011. Only patients diagnosed with AIP and IgG4 disease based on accepted international criteria were included in the study. ${ }^{1}$

Results 16 patients were identified during this period. Mean age group was 64 years (Range 43-83 yrs). Male=15, Female=1. Abnormal LFTs were present in $62.5 \%$ of patients. Mean bilirubin was $97 \mathrm{mmols} / \mathrm{l}$ (range 4-354). Mean total IgG was $15.9 \mathrm{~g} / \mathrm{l}$ (range 7.5-26.7). Mean IgG4 subclass levels was $6.4 \mathrm{~g} / \mathrm{l}$ (Range 0.27-24.6). Pancreas was affected in $88 \%$ (15) and biliary abnormalities were seen in $62.5 \%(10)$ of the cases. Other organs noted to be involved were pericardium (1), retroperitoneum (2), gall bladder (2). Two patients had duodenal obstruction due to inflammation of the duodenum, stomach, peripancreatic area and the gall bladder bed. CT scan revealed enlarged head of pancreas (HOP) in nine patients $(57 \%)$, extra-pancreatic mass in $4(25 \%)$, extra hepatic bile duct involvement in $10(62.5 \%)$ and intrahepatic bile duct involvement in 9 (37.5\%). 3 (18.75\%) patients underwent ERCP and two had stenting of biliary strictures. EUS was performed in nine patients-showed enlarged HOP in $4(44 \%)$ and changes of chronic pancreatitis-4 (44\%). Nine patients (56\%) had a raised serum IgG4. Diagnosis was made at surgery or by laparoscopic biopsy in 7 (44\%) patients. Final diagnosis: Type I AIP in 15 patients (88\%), Type II AIP in $1(6 \%)$ and IgG4 cholangiopathy with no pancreatic involvement in $1(6 \%)$. Other autoimmune diseases that were associated were Raynauds disease (1) and Sjogren's syndrome (1). Steroids were initiated in $12(75 \%)$ patients (mean dose $37.5 \mathrm{mg}$ ). Disease relapsed in three patients (25\%). Azathioprine was started on five patients. One patient was switched to 6MP due to side effects.

Conclusion Extrapancreatic disease, especially biliary structuring appears to be common. As this condition mimics malignancy, a combination of modalities were needed to arrive at a diagnosis. Relapse in not uncommon and a small group of patients will require additional immunosuppression for control of the disease.

Competing interests None declared.

\section{REFERENCE}

1. Okazaki K, Kawa S, Kamisawa $T$, et al. Clinical diagnostic criteria of autoimmune pancreatitis: revised proposal. J Gastroenterol 2006;41:626-31.

\section{PMO-110 AN AUDIT OF PANCREATIC CANCER OUTCOMES IN THE DISTRICT GENERAL HOSPITAL SETTING: OUTCOMES APPEAR BETTER THAN NATIONAL AVERAGE}

doi:10.1136/gutjnl-2012-302514b.110

${ }^{1} \mathrm{~T}$ Akbar, ${ }^{*}{ }^{2} \mathrm{~F}$ Murphy. ${ }^{1}$ Department of Gastroenterology, Royal Hampshire County Hospital, Winchester, UK; ${ }^{2}$ Royal Hampshire County Hospital, Winchester, UK

Introduction Pancreatic cancer outcomes nationally demonstrate poor survival outcomes with 1 and 3-month survival rates of $73 \%$ and $47 \%$. We performed an audit of our experience of our patients with pancreatic cancer in the setting of a district general hospital and compared our results with the national dataset.

Methods We carried out data search from our system with all patients with a new diagnosis over 16 months from June 2010 to October 2011. The information was correlated with case notes and relevant histology and radiology reports. The data were collected and entered on an Excel spreadsheet for analysis.

Results 21 new diagnoses with a mean age of 73, range from 56 to 95. The male to female ratio was $3: 2$. The most common presenting symptoms were jaundice and abdominal pain. The average duration of symptoms developing to being seen was approximately 70 days. Patients on average had a CT scan within 7 days of being seen in clinic. Range 1-29 days. The most common radiological diagnosis was head of the pancreas tumour; representing $52 \%$ of cases. $62 \%$ of patients had biliary obstruction of which $69 \%$ had an ERCP and $31 \%$ had PTC insertion of a stent. The stent was changed at least once in $38 \%$ of these patients. $14 \%$ of patients had duodenal obstruction. 19\% of patients underwent Whipples procedure with $57 \%$ patients having chemotherapy. Gemcitabine/Cisplatin combination was most commonly used. $48 \%$ of patients had died with a mean of 154 days from when they were first seen. The range was $80-226$ days. $75 \%$ of the patients who had Whipples procedure and post op chemotherapy had metastatic disease on CT on average 10 months later. Of those that are still alive, only one other than those that had the Whipples procedure had survived more than a year.

Conclusion Our data demonstrated that our outcomes were better than the national data set produced by the Pancreatic Cancer UK charity. The average 1-year survival is $16 \%$ and our figures show that $24 \%$ of our patients survived more than 1-year. Our cumulative survival at 1-month and three months was comparable to the national average (100\% and $86 \%$ vs $74 \%$ and $47 \%$ ), are patients have radiological diagnosis sooner and more of our patients go on to have surgery. Our survival data supports that as a district general hospital we are able to manage patients' pancreatic cancer effectively and safely with good outcomes.

Competing interests None declared.

\section{PM0-111 SHOULD ALL PATIENTS WITH LOCALLY ADVANCED PANCREATIC CANCER BE OFFERED INTRAOPERATIVE ASSESSMENT?}

doi:10.1136/gutjnl-2012-302514b.111

${ }^{1} \mathrm{~V}$ Rao, ${ }^{*}{ }^{1} \mathrm{~K}$ Chantladze, ${ }^{1} \mathrm{~S}$ Kugathasan, ${ }^{2} \mathrm{O}$ Byass, ${ }^{2} \mathrm{~A}$ Razack, ${ }^{1} \mathrm{~K}$ Wedgwood. ${ }^{1}$ Department of Surgery, Castle Hill Hospital, Cottingham, UK; ${ }^{2}$ Castle Hill Hospital, Cottingham, UK

Introduction Surgery is the only potentially curative option in patients with pancreatic cancer. Hence it is extremely important that the diagnostic tests used to ascertain resectability is very reliable before this curative option is denied to this unfortunate group of patients. CT and Endoscopic Ultrasound (EUS) which are commonly used as part of pre operative staging was compared with intraoperative findings to assess diagnostic reliability in determining resectability in patients with pancreatic cancer. 